\title{
Erratum to: Geometrical Properties and Turbulent Flame Speed Measurements in Stationary Premixed V-flames Using Direct Numerical Simulation
}

\section{Thomas D. Dunstan • Nedunchezhian Swaminathan •}

Ken N. C. Bray • R. Stewart Cant

Published online: 5 October 2010

(C) Springer Science+Business Media B.V. 2010

Erratum to: Flow Turbulence Combust (2011) 87:237-259

DOI 10.1007/s10494-010-9284-1

In Section 3.4 incorrect values of the turbulence energy dissipation rate, $\tilde{\epsilon}$, and the Karlovitz number, Ka, were used in the evaluation of Eqs. 27, 28 and 30. The errors affect the results shown in Fig. 10, and some of the conclusions in Section 4.

Equations 27, 28, and 30 in the original paper are respectively:

$$
s_{T}^{k p p}=2 \sqrt{\frac{v_{t}}{\rho_{0} \mathrm{Sc}}\left(\frac{\partial \overline{\dot{\omega}}}{\partial \tilde{c}}\right)_{\tilde{c} \rightarrow 0}}
$$

where $v_{t}$ is the turbulent viscosity $v_{t}=C_{\mu}\left(\tilde{k}^{2} / \tilde{\epsilon}\right)$, and $\tilde{k}$ and $\tilde{\epsilon}$ are the Favre turbulent kinetic energy and dissipation rate.

$$
\begin{gathered}
\frac{s_{T}^{m}}{s_{L}}=\left\{\frac{18 C_{\mu}}{\left(2 C_{m}-1\right) \beta^{\prime}}\left[\left(2 K_{c}^{*}-\tau C_{4}\right)\left(\frac{u^{\prime} \Lambda}{s_{L} \delta_{t h}}\right)+\frac{2 C_{3}}{3}\left(\frac{u^{\prime}}{s_{L}}\right)^{2}\right]\right\}^{\frac{1}{2}} \\
\frac{s_{T}^{m *}}{s_{L}}=\left\{\frac{u^{\prime} \Lambda 18 C_{\mu}+8 \widetilde{D}_{c}}{\left(2 C_{m}-1\right) \beta^{\prime}}\left[\frac{\left(2 K_{c}^{*}-\tau C_{4}\right)}{s_{L} \delta_{t h}}+\frac{2 u^{\prime} C_{3}}{3 \Lambda s_{L}^{2}}\right]\right\}^{\frac{1}{2}}
\end{gathered}
$$

The online version of the original article can be found at http://dx.doi.org/10.1007/s10494-010-9284-1.

T. D. Dunstan $(\varangle) \cdot$ N. Swaminathan · K. N. C. Bray · R. S. Cant

Department of Engineering, University of Cambridge, Trumpington Street,

Cambridge, CB2 1PZ, UK

e-mail: tdd25@cam.ac.uk 
where $C_{4}=1.1(1+\mathrm{Ka})^{-0.4}, C_{3}=1.5 \sqrt{\mathrm{Ka}} /(1+\sqrt{\mathrm{Ka}})$, and $\mathrm{Ka}$ is the local Karlovitz number.

The Karlovitz number used in the original paper, $\widehat{\mathrm{Ka}}$, was based on the thermal laminar flame thickness $\delta_{t h}$, however, model constants used in Eqs. 28 and 30 were derived using the diffusive flame thickness, $\delta=\delta_{t h} / 2(1+\tau)^{0.7}$. This results in an overestimation of the Karlovitz number by $\widehat{\mathrm{Ka}} \simeq 2.2 \mathrm{Ka}$.

Additionally, a miscalculation of the dissipation rate $\tilde{\epsilon}$ by a factor of two, introduced a second error to the uncorrected Karlovitz number of $\Delta \widehat{\mathrm{Ka}} / \widehat{\mathrm{Ka}}=0.5 \Delta \tilde{\epsilon} / \tilde{\epsilon}$. The effects of these errors are similar in magnitude and can be most clearly seen at the highest turbulence intensity Case III. Figure 1 below gives the original and revised calculations for $s_{T}^{m *}$ (Eq. 30, which is Eq. 3 above) for Case III. The effects of individually applied corrections for $\mathrm{Ka}$ and $\tilde{\epsilon}$ are also shown. In all cases the effect of the corrections is to increase the model flame speed predictions, and improve the agreement between the modelled and actual values.

Figure 2 below is the revised version of Fig. 10 in the original article. The consumption speed data has been omitted since this remains unchanged from the original.

In light of these revised figures the following points should be noted:

- In contrast to the original analysis, it appears that the modified model of Eq. 30 provides a good estimate of the displacement speed of the flame brush leading edge without the need to correct for mean tangential convection as described by Eq. 31. This lends support to the underlying premise of scalar dissipation rate (SDR) modelling, in that the dynamics of the SDR are governed by the smallest scales of the flow and are essentially independent of the mean flow geometry.

- The agreement between the unmodified model $s_{T}^{m}$ and the KPP estimate $s_{T}^{k p p}$ for Case III is not now as close as it originally appeared. However, as noted in Section 3.1, the statistical convergence of the mean flow variables in Case III is not as good as for cases I and II, and this inevitably leads to greater variation in the derivative $\partial \overline{\dot{\omega}} / \partial \tilde{c}$ in Eq. 27 . The subsequent analysis and conclusions are not altered by this.

Fig. 1 Corrected and uncorrected model turbulent flame speeds (Eq. 30) compared to actual values as a function of downstream position for Case III, evaluated on the $\tilde{c}=0.05$ isoline

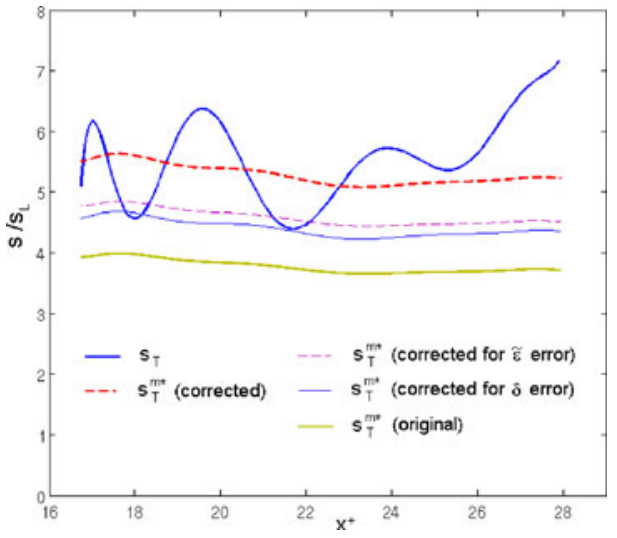



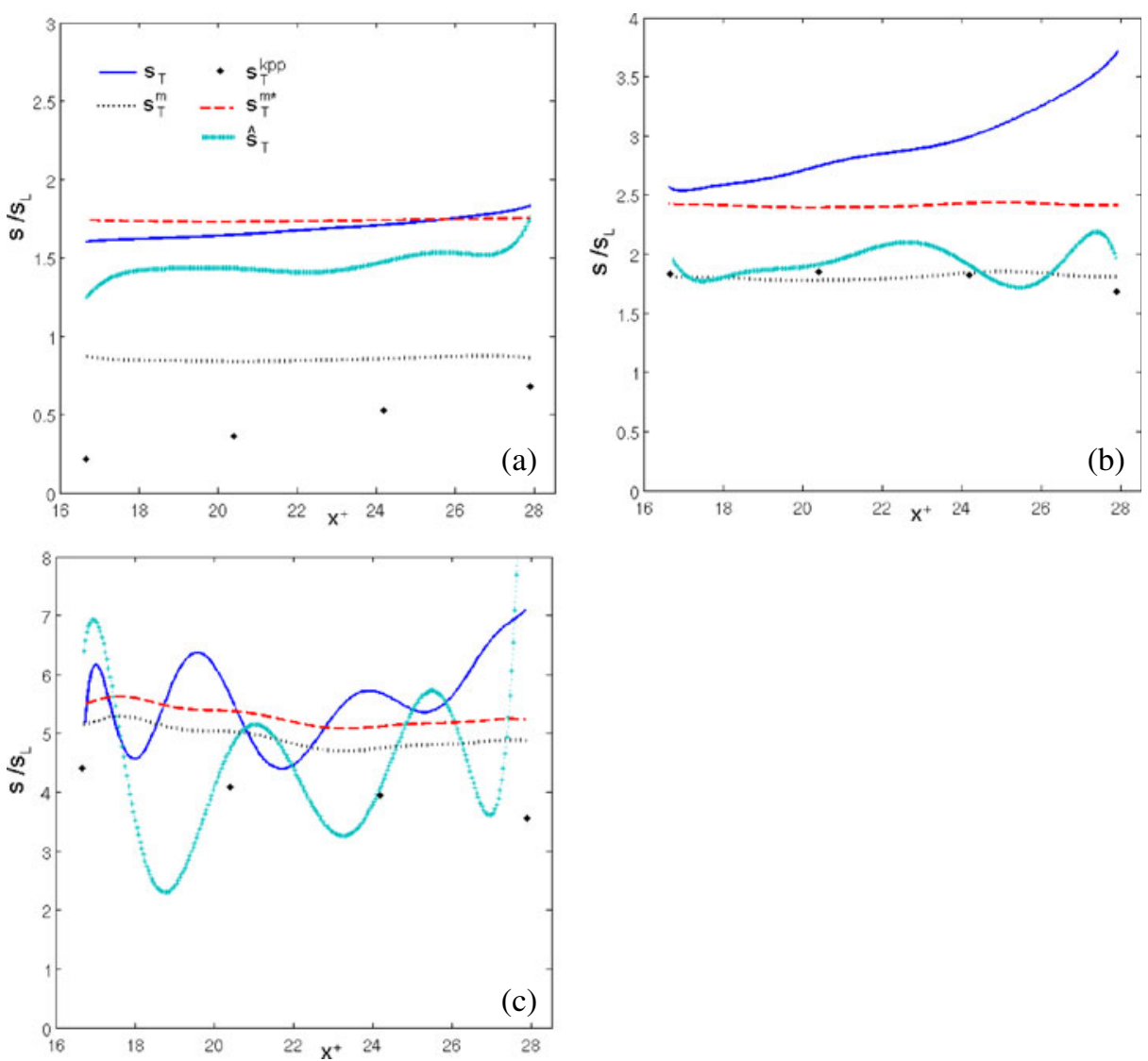

Fig. 2 Turbulent flame speeds as a function of downstream position for Cases I-III (a-c) evaluated on the $\tilde{c}=0.05$ isoline. Point estimates of $s_{T}^{k p p} / s_{L}$ are shown as solid symbols for positions 1-4 (Fig. 4)

Cases I and II remain relatively unchanged compared to Case III, except to note that the unmodified flame speed model of Eq. 27 is now much closer to the actual values than originally suggested.

- In Section 4 the penultimate paragraph should now read:

The flame speed predictions of a recently developed model by Kolla et al. [29], based on scalar dissipation rate modelling combined with a flamelet assumption and a KPP analysis, were also assessed. The omission of mean molecular diffusion terms in the model resulted in large discrepancies at the lower turbulence intensities. Incorporating molecular diffusion into a modified model improved the accuracy across all cases. A pseudopropagation speed was defined in which the convective flux of $\tilde{c}$ tangential to the mean flame normal was subtracted from the total, in order to assess the importance of the planar flame assumption in the model derivation. Agreement with the model was not improved compared to the unadjusted 
propagation speed $s_{T}$, suggesting that the effects of the additional flux may be implicitly taken into account through the existing model parameters. Further work on this is ongoing, but the general agreement between the modelled and measured values demonstrates the applicability of the KPP analysis and scalar dissipation rate modelling approach to all but the lowest turbulence Reynolds numbers considered. 